\title{
Coherent and incoherent metamaterials and the order-disorder transitions
}

\author{
N. Papasimakis, ${ }^{1}$ V. A. Fedotov, ${ }^{1}$ Yuan Hsing Fu, ${ }^{1,2}$ Din Ping Tsai, ${ }^{2}$ and N. I. Zheludev ${ }^{1, *}$ \\ ${ }^{1}$ Optoelectronics Research Centre, University of Southampton, SO17 1BJ, UK \\ ${ }^{2}$ Department of Physics, National Taiwan University, Taipei 1061\%, Taiwan
}

(Dated: May 4, 2019)

\begin{abstract}
We demonstrate a new class of "coherent" metamaterials, where a regular ensemble of metamolecules shows a collective, i.e. coherent, narrow band resonant response, while disordering the ensemble leads to broadening and eventually disappearance of the resonance. We draw parallels between the observed collective behavior of meta-molecules and the Mössbauer effect and notice certain remarkable similarities with the phase transitions of ferromagnetic systems.
\end{abstract}

Artificial electromagnetic metamaterials provide a uniquely fertile ground for achieving all kinds of unusual functionalities: they show a negative index of refraction required for the creation of diffraction-free superlenses [1, exhibit strong optical magnetism [2, 3] and impose asymmetric transmission of light [4]. Metamaterial structures can be invisible [5], act as electromagnetic cloaks [6, 7, show exceptionally high or zero refractive indices [8] and even behave like optical frequency "superconductors" repelling the magnetic field of the optical wave, thus mimicking the Meissner effect [9. The recent development of self-assembly techniques for fabrication of metamaterials that yield randomized arrays of meta-molecules [10] and new ideas for using metamaterials in coherent sources of electromagnetic radiation [11] stimulated our interest in the effects of positional disorder on the electromagnetic properties of two-dimensional metamaterial arrays. In this work we have identified two distinctively different classes of artificial structures, the "coherent" and "incoherent" metamaterials with narrow-band resonant spectral response. The response of incoherent metamaterials is essentially determined by the properties of the individual meta-molecules and is virtually insensitive to positional disorder. In "coherent" metamaterials, external electromagnetic excitation induces a magnetic response in individual meta-molecules. Here the metamolecular disorder dramatically modifies the spectral response and the magnetization of the structure exhibits a collective, i.e. "coherent" nature underpinned by interactions between the magnetic moments of the metamolecules.

Metallic ring meta-molecules are probably the most popular building blocks of metamaterial structures. They are employed to provide a strong magnetic response with negative permeability, required for achieving a negative index of refraction. For this purpose, both the ring and the wave propagation direction lie on the same plane, so that the circular current induced on the ring creates a magnetic moment parallel to the magnetic field of the incident wave. Here we investigate two novel types of metallic planar ring metamaterials supporting electromagnetic modes with high quality factors (see Fig. 1), where the magnetic moments associated with the in- duced currents are perpendicular to the plane of the array. Meta-molecules of the first type were formed by pairs of concentric metal rings, while meta-molecules of the second type had the form of asymmetrically-split rings. In both types of metamaterials studied in this work, the incident wave is normal to the plane of the structure and therefore the interaction of the induced magnetic moments with the magnetic field of the incident wave is negligible. This ensures a key role of inter-meta-molecular interactions in the formation of the electromagnetic response of the material.

A special feature of the studied structures is that they support high-quality resonances associated with antisymmetric currents. In the asymmetrically split ring, these currents flow in the longer and shorter arc of the ring, while in the concentric rings they oscillate in the neighboring sections of the two rings (see insets to Figs. 1a \& 1b). In both cases, the far-field electric dipole radiation is cancelled due to the induced counterpropagating currents, ensuring low radiation losses and thus high-Q factors of the resonances [12. Figure 1 shows

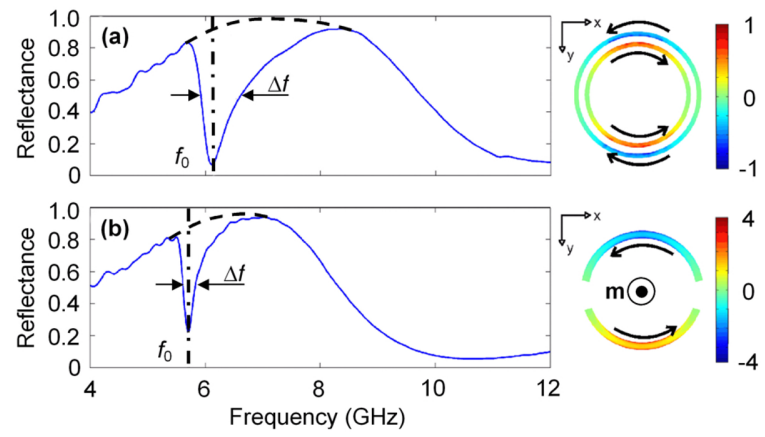

FIG. 1: Normal incidence reflectivity spectra of regular metamaterial arrays composed of pairs of concentric rings (a) and asymmetrically-split rings (b), measured. The insets show the induced anti-symmetric current modes corresponding to the reflectance minima. In the case of the asymmetricallysplit rings, the anti-symmetric current configuration leads to a magnetic dipole moment $(m)$ normal to the plane of the rings. On the contrary, concentric rings do not exhibit a magnetic moment at resonance. 
experimentally measured reflectance spectra of regular two-dimensional arrays for both types of meta-molecules. The radius of the asymmetrically-split ring structure was $6 \mathrm{~mm}$ and the line thickness $0.8 \mathrm{~mm}$, while the length of the arcs corresponded to angles of 160 and 140 degrees. The radii of inner and outer rings forming pairs of the concentric rings were $4.5 \mathrm{~mm}$ and $5.45 \mathrm{~mm}$ respectively and the line thickness of each ring was $0.4 \mathrm{~mm}$. The unit cells of the regular structures had the size of $15 \times 15 \mathrm{~mm}$, which ensured no diffraction at frequencies below $20 \mathrm{GHz}$. In each case, the reflectivity spectrum features a broad background of high reflection that is split by a narrow resonant dip associated with highquality anti-symmetric current modes.

Despite the obvious similarity in the origin of the narrow resonant response of the two metamaterials, their behavior becomes dramatically different upon disordering the regular meta-molecule lattice. The effect of disorder was studied experimentally by introducing random displacements of the ring meta-molecules from the perfect double-periodic grid according to a random uniform distribution defined in the square interval $x \epsilon(-\alpha / 2, \alpha / 2), y \epsilon(-\alpha / 2, \alpha / 2)$, while all other parameters of the structures were fixed (see representative cases in Fig. 2). A disorder parameter, $D$, was defined as the ratio of $\alpha$ over the unit cell side. The evolution of the reflectance spectra with increasing disorder is presented in Fig. 3. For the concentric ring metamaterial, the resonant dip in the reflectivity robustly retains its magnitude and width even for very high levels of disorder, which may be seen as the persistence of the blue band centered at $6.2 \mathrm{GHz}$. For the asymmetrically-split ring metamaterial, however, the similar resonant dip at $5.7 \mathrm{GHz}$ degrades rapidly and completely vanishes for a moderate degree of disorder. This is further illustrated in Fig. 3 with two characteristic cross-sections of the spectral evolution corresponding to cases of weak $\left(D_{1}=0.07\right)$ and moderate $\left(D_{2}=0.40\right)$ disorder.

The dramatic difference in the behavior of the two metamaterials becomes apparent, when the dependence of their resonance quality factors on the disorder is con-

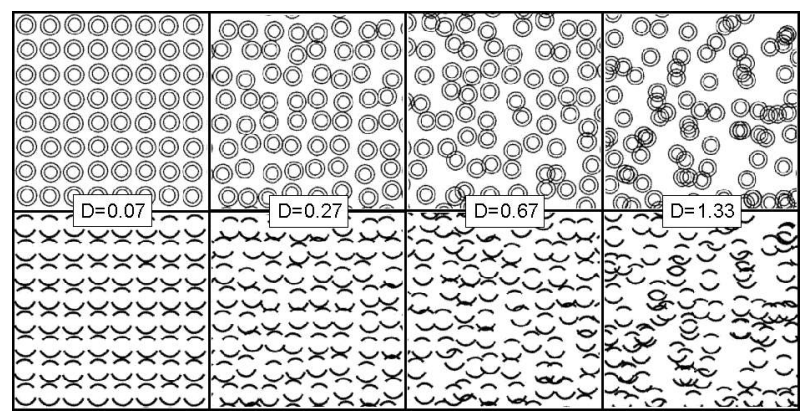

FIG. 2: Fragments of disordered metamaterial samples. The disorder parameter, $D$, increases from left to right.
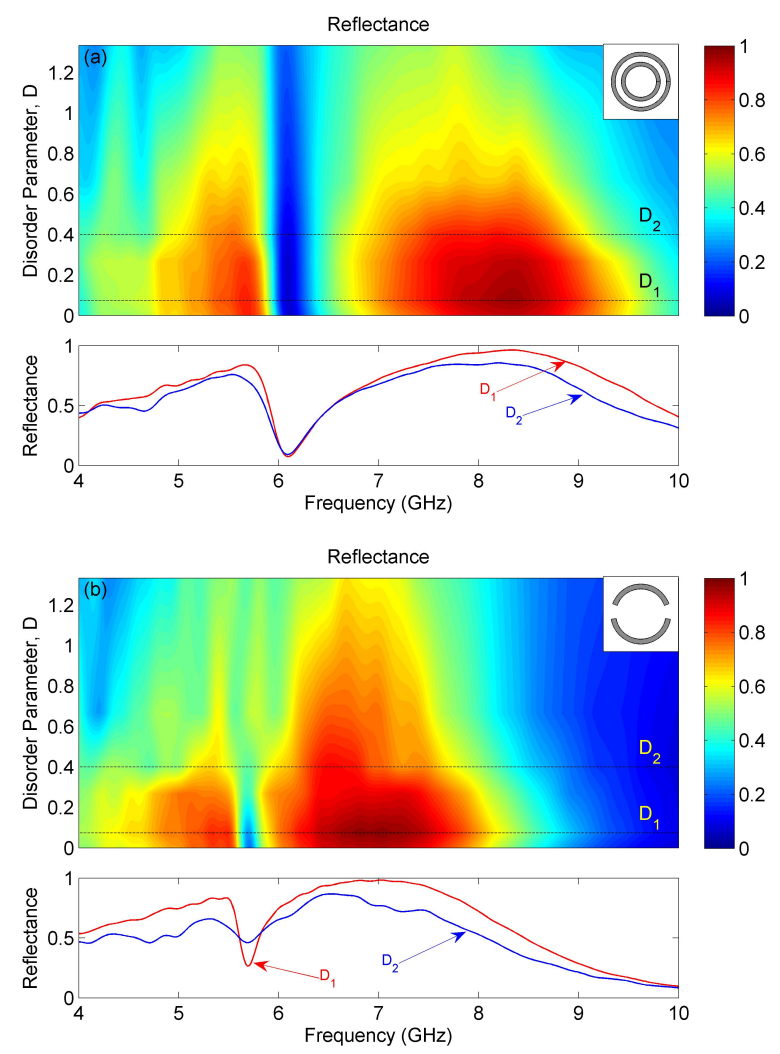

FIG. 3: Color maps (a) and (b) present the reflectance as a function of frequency and disorder parameter $D$ for the arrays of correspondingly concentric and asymmetrically-split rings. The two marked cross-sections show reflectance profile at $D_{1}=0.07$ and $D_{2}=0.40$, respectively.

sidered (see Fig. 4). Here the Q-factor represents directly the average strength of the anti-symmetric component of the current mode induced in the meta-molecules by the incident electromagnetic wave. If individual metamolecules do not interact and thus do not form a collective, coherent mode, disorder will not affect the spectral lines of the structure. This is well illustrated by the behavior of the concentric split ring metamaterial, which we classify as an "incoherent" metamaterial. On the other hand, if individual meta-molecules show narrow spectral lines that are affected by inter-molecular interactions, the spectral lines will inhomogeneously broaden as a result of randomization of the inter-molecular distances and thus of the inter-molecular interactions affecting the lines (spectral shift and split). This is a trivial case that is not relevant to the "coherent" metamaterial behavior considered here. In "coherent" metamaterials, narrow lines are not seen in individual meta-molecules and appear only in ordered arrays. Here randomization leads to radiation damping of the collective, coherent response of the array. We will see below that an ensemble of asymmetrically-split rings belongs to this category of "coherent" metamaterials. 


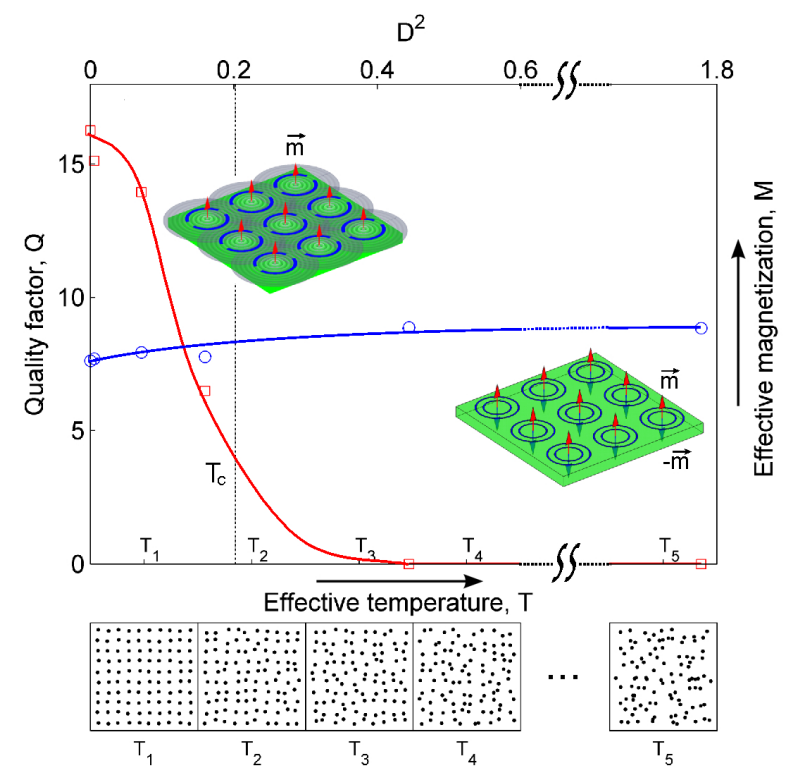

FIG. 4: Resonance quality factor of the metamaterials' response and effective magnetization as a function of the disorder parameter squared (effective temperature) plotted for arrays of concentric (blue) and asymmetrically-split (red) rings. Points correspond to experimentally measured values of $Q$, while lines serve as guides for the reader. The rapid decay of the effective magnetization associated with the split-rings' response allows the introduction of a critical temperature $T_{c}$ similar to the Curie temperature in ferromagnetics. The insets illustrate the nature of interactions between the metamolecules. A sequence of frames with dot arrays illustrates the increase of disorder in the positions of the meta-molecules with increasing $D^{2}$ (effective temperature of the system).

In an ensemble of concentric rings, the Q-factor of the resonant line is practically independent of disorder. This is due to the fact that, at resonance, the anti-symmetric currents of the inner and outer rings create magnetic moments pointing in opposite directions, thus rendering the total magnetic response of the meta-molecules extremely weak. Furthermore, by design, the resonant electric response of the concentring rings is also very weak (which ensures high Q-factors). Therefore, mutual interactions between the meta-molecules (both of electric or magnetic dipole-type) are negligible, and the collective response of the arrays does not depend on the positions (or disorder) of the molecules, thus reducing to a direct sum of the individual molecular contributions. This experimental result is further illustrated by our numerical simulations presented in Fig. 5, where it is shown that an anti-symmetric current mode leading to a high Q-factor resonant response can be excited even in a single isolated concentric-ring meta-molecule. Negligible magnetization renders the magnetic behavior of this type of planar metamaterial similar to that of a diamagnetic gas of atoms with paired electrons and thus represents an example of an ideal diamagnetic metamaterial.
On the contrary, in the case of asymmetrically-split ring arrays, the Q-factor appears to be extremely sensitive to disorder. At the resonance, the currents induced in the upper and lower arcs of the split-rings oscillate in opposite phases and therefore create coherent magnetic dipole moments oscillating in phase. These magnetic moments are oriented normal to the plane of the array. In a regular array, interference of waves re-radiated by the oscillating magnetic dipoles results in the so-called magneto-inductive waves [13, which are confined to the plane of the array mediating efficiently strong interactions between the meta-molecules (see illustration in the inset to Fig. 4). The increase of disorder leads to intense scattering of these waves into free space (conversion into free space electromagnetic waves) and, consequently, to losses that reduce the strength of the anti-symmetric current mode. Consequently, the inter-molecular interactions become weaker resulting in a lower Q-factor and vanishing net magnetization. Importantly, in the limiting case of a single isolated asymmetrically-split ring, the magnetic dipole scattering is at maximum and the high-Q resonance is completely absent, as opposed to a pair of concentric rings (see Fig. 5). Thus, the broadening/weakening of the collective response cannot be explained by splitting or shifting of the high-Q resonances of the individual meta-molecules that form clusters of closely spaced or even overlapping split rings in the disordered arrays, as the resonance simply does not exist for an individual meta-molecule. Note, that the Q-factor of the metamaterial's response here is not only a direct measure of the strength of the anti-symmetric current mode, but also a measure of the metamaterial's effective magnetization, induced normal to the plane of the array by the incident electromagnetic wave.

Intriguingly, the rapid decay of the effective magnetization with increasing disorder resembles phase transitions in magnetic solid-state systems, such as the behavior of a two-dimensional array of spins (Ising ferromagnet [14]) in the absence of external fields with increasing temperature. This similarity is not coincidental and reflects a common physical origin. In the Ising model the Hamiltonian of the system is given by $H=\Sigma J_{i j} S_{i} S_{j}$, where $J_{i j}$ is a constant that reflects the strength of interaction between spins $S_{i}$ and $S_{j}$. With increasing temperature, the ratio of the interaction energy $J$ over the energy of thermal fluctuations $\left(k_{\mathrm{B}} T\right)$ decreases. This leads to a secondorder phase transition from the initial ferromagnetic ordered state, where all the spins pointed in the same direction, to a diamagnetic disordered state with random spin orientations, corresponding to a vanishing macroscopic magnetization. In the ensemble of asymmetricallysplit rings, the induced magnetic moments $m$ of the individual meta-molecules interact in a similar way and therefore the system's Hamiltonian will have the following form: $H=<\Sigma j_{i j} m_{i} m_{j}>$, where brackets indicate time average over one period of the incident wave and no 
coupling exists between the oscillating magnetic dipoles and external magnetic field due to their orthogonality. Here the transition to the diamagnetic state results from the destruction of the coherent surface state by scattering. Furthermore, by considering random displacements of meta-molecules in the metamaterial arrays as a result of 'heating' (similar to real crystalline solids) the disorder parameter can be related to an effective temperature as follows, $T \sim D^{2}$. Although the analogy between the asymmetrically-split ring system and Ising ferromagnet is phenomenological, direct mapping into more complicated spin systems, such as spin glasses [15] is also possible.
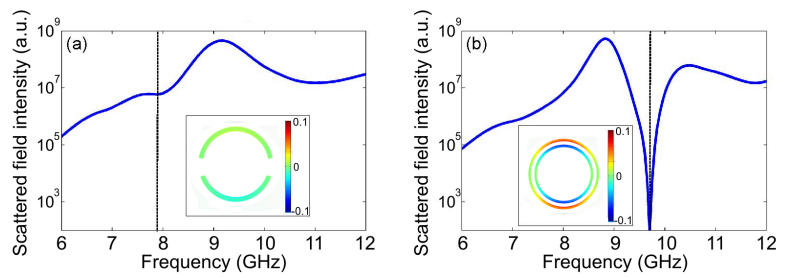

FIG. 5: (a) Scattered intensity for a single asymmetrically split ring (ASR) and (b) for a single concentric ring structure. The dashed lines mark the position of the antisymmetric current configuration for each case. Color maps of the associated current modes (projection along the horizontal axis) are provided in the insets.

The loss of coherency by disorder presented here is fundamentally different from the recently investigated inhomogeneous line broadening in disordered photonics crystals [16 and metamaterials [17, 18 that were concerned with negative permeability associated with "incoherent" response due to random changes in the geometry of the individual meta-molecules. On the contrary, in the "coherent" metamaterial described here, the sharp spectral feature is a collective resonance of the entire ordered structure. This behavior is reminiscent of another well known phenomenon in many-body physics, namely the Mössbauer effect [19], where transitions of atomic nuclei with extremely narrow line-widths can only be observed when the atoms form a crystal lattice, while in an atomic gas the emission line is strongly broadened by the recoil during the emission of the high energy gamma quanta. In a similar way, a single meta-molecule of the coherent metamaterial does not exhibit the narrow resonance associated with the antisymmetric current mode (also known as trapped-mode resonance) as a result of scattering losses, while in a regular array no scattering losses are possible for wavelengths longer than the array pitch and thus a low-frequency high quality mode is formed.

The two planar metamaterial structures considered above are examples of artificial media with strong and weak inter-meta-molecular interactions representing two characteristic and antipode classes of what we call "coherent" and "incoherent" metamaterials. The fundamen- tal difference in the nature of their narrow resonances, collective in the first case and individual in the second, determines the potential applications of these structures, most notably the lasing spaser [11. The lasing spaser, a metamaterial analog of spaser [20] is a planar narrow-diversion coherent source of electromagnetic radiation that is fuelled by plasmonic oscillations of a twodimensional resonator array. Here the coherency of the optical source is ensured by the synchronous oscillations of the plasmonic currents in the array. In a "coherent" metamaterial formed by asymmetrically-split rings, the regular array gives the highest value of quality factor compared to disordered arrays. We argue that, similarly, when both phased (coherent) and uncorrelated (incoherent) current oscillations are present in an array of metamolecules of this type, the uncorrelated component will decay more rapidly. Therefore in the presence of gain the phased, coherent component of amplified spontaneous current fluctuations will win over incoherent fluctuations providing for a self-starting regime of the lasing spaser. On the contrary, "incoherent" metamaterials with weak inter-molecular interactions, such as concentric rings, do not possess a mechanism of synchronization of current oscillation in individual meta-molecules and are not suitable for lasing spaser applications. However,the resonant properties of "incoherent" metamaterials are more tolerant to disorder making them more suitable for manufacturing using methods prawn to imperfections such as self-assembly [10].

Finally, another intriguing and unique property of the "coherent" metamaterials will be the dependence of their transmission and reflection spectra on the size of the sample array. As has been demonstrated by numerical calculations narrow band resonance is only featured in the spectrum when a large number of meta-molecules are involved in the formation of the optical response. We argue that in a similar way optical spectra measured with spatially coherent and incoherent sources of electromagnetic radiation will be different, with sharp resonances featured only under coherent illumination.

The authors are grateful to Peter Nordlander for stimulating discussions and guidance on the electromagnetic response of disordered systems. This work is supported by the European Union through the FP7 ENSEMBLE project and by the Engineering and Physical Sciences Research Council (UK) through the Nanophotonics Portfolio Grant and the International Collaborative grant with National Taiwan University.

* Electronic address: niz@orc.soton.ac.uk URL: ww. nanophotonics.org.uk/niz

[1] D. R. Smith, J. B. Pendry, and M. C. K. Wiltshire, Science 305, 788 (2004).

[2] C. Enkrich et al., Phys. Rev. Lett. 95, 203901 (2005). 
[3] W. Cai et al., Opt. Express 15, 3333 (2007).

[4] A. S. Schwanecke et al., Nano Letters 8, 2940 (2008).

[5] V. A. Fedotov et al., Phys. Rev. E 72, 056613 (2005).

[6] J. B. Pendry, D. Shurig and D. R. Smith, Science 312, 1780 (2006).

[7] U. Leonhardt, Science 312, 1777 (2006).

[8] V. A. Fedotov et al., Appl. Phys. Lett. 88, 091119 (2006).

[9] A. S. Schwanecke et al., J. Opt. A: Pure Appl. Opt. 9, L1 (2007).

[10] D. A. M. Pawlak, Scientia Plena 4, 014801 (2008).

[11] N. I. Zheludev et al., Nature Photon. 2, 351 (2008).

[12] V. A. Fedotov et al., Phys. Rev. Lett. 99, 147401 (2007).
[13] E. Shamonina et al., J. Appl. Phys. 92, 6252 (2002).

[14] E. Ising, Z. Physik 31, 253 (1925).

[15] D. Sherrington and S. Kirkpatrick, Phys. Rev. Lett. 35, 1792 (1975).

[16] D. Nau et al., Phys. Rev. Lett. 98, 133902 (2007).

[17] M. V. Gorkunov et al., Phys. Rev. E 73, 056605 (2006).

[18] J. Gollub et al., Appl. Phys. Lett. 91, 162907 (2007).

[19] R. L. Mőssbauer, Z. Physik A 151, 124 (1958).

[20] D. J. Bergman and M. I. Stockman, Phys. Rev. Lett. 90, 027402 (2003) 